ACUTE LUNG INJURY

\title{
Regulation of vascular endothelial growth factor bioactivity in patients with acute lung injury
}

\author{
G D Perkins, J Roberts, D F McAuley, L Armstrong, A Millar, F Gao, D R Thickett
}

Thorax 2005;60:153-158. doi: 10.1136/thx.2004.027912

See end of article for authors' affiliations

Correspondence to:

Dr D Thickett, Lung Injury

Fibrosis Treatment

Programme, University of Birmingham, Lung Investigation Unit, Nuffield House, Queen Elizabeth Hospital, Birmingham B15 2TT, UK; d.thicket@@ bham.ac.uk

Received 7 May 2004 Accepted 23 November 2004
Background: Reduced bioactive vascular endothelial growth factor (VEGF) has been demonstrated in several inflammatory lung conditions including the acute respiratory distress syndrome (ARDS). sVEGFR-1, a soluble form of VEGF-1 receptor, is a potent natural inhibitor of VEGF. We hypothesised that sVEGFR-1 plays an important role in the regulation of the bioactivity of VEGF within the lung in patients with ARDS. Methods: Forty one patients with ARDS, 12 at risk of developing ARDS, and 16 normal controls were studied. Bioactive VEGF, total VEGF, and sVEGFR-1 were measured by ELISA in plasma and bronchoalveolar lavage (BAL) fluid. Reverse transcriptase polymerase chain reaction for sVEGFR-1 was performed on BAL cells.

Results: sVEGFR-1 was detectable in the BAL fluid of $48 \%(20 / 41)$ of patients with early ARDS (1.4$54.8 \mathrm{ng} / \mathrm{ml}$ epithelial lining fluid (ELF)) compared with $8 \%(1 / 12)$ at risk patients $(p=0.017)$ and none of the normal controls $(p=0.002)$. By day 4 sVEGFR- 1 was detectable in only $2 / 18$ ARDS patients $(p=0.008)$. Patients with detectable sVEGFR-1 had lower ELF median (IQR) levels of bioactive VEGF than those without detectable sVEGFR-1 (1415.2 (474.9-3192) pg/ml v 4761 (1349-7596.6) pg/ml, median difference $3346 \mathrm{pg} / \mathrm{ml}(95 \% \mathrm{Cl} 305.1$ to 14711.9$), \mathrm{p}=0.016)$, but there was no difference in total VEGF levels. BAL cells expressed mRNA for sVEGFR-1 and produced sVEGFR-1 protein which increased following incubation with tumour necrosis factor $\alpha$.

Conclusion: This study shows for the first time the presence of sVEGFR-1 in the BAL fluid of patients with ARDS. This may explain the presence of reduced bioactive VEGF in patients early in the course of ARDS. l: order to preserve gas exchange, the lung needs a confluent layer of alveolar epithelial and endothelial cells to prevent flooding of the alveolar space with fluid and plasma proteins. When this layer is damaged the resultant non-cardiogenic pulmonary oedema leads to refractory hypoxia and the need for mechanical ventilation. Vascular endothelial growth factor (VEGF) plays a central role in embryonic pulmonary angiogenesis and development. ${ }^{1}$ In human fetuses at term, low alveolar levels of VEGF are associated with the development of a severe respiratory distress syndrome. ${ }^{2}$ As the lung develops VEGF protein levels within the alveolus increase such that, by adulthood, the epithelial lining fluid (ELF) of normal adults has $11 \mathrm{ng} / \mathrm{ml}$ of VEGF protein. ${ }^{2}$ This is 500 times higher than the average plasma level. ${ }^{2}$ In this regard the lung is almost unique, since most tissues express much lower levels of VEGF. In situ hybridisation has shown that the major sources of VEGF in the lung are mesenchymal and type II alveolar cells. ${ }^{2}$ During acute inflammation alveolar macrophages and neutrophils may also play a role in VEGF production. ${ }^{3}$

The function and regulation of VEGF in acute lung injury (ALI) and acute respiratory distress syndrome (ARDS) is complex. VEGF is a potent endothelial cell mitogen and permeability factor. Clinical studies have reported increased plasma VEGF levels in a wide variety of inflammatory conditions where increased capillary permeability is a feature such as pre-eclampsia, ${ }^{4}$ inflammatory pleural effusion, ${ }^{5}$ and ARDS. ${ }^{6}$ In vitro studies using plasma from patients with ARDS showed that the addition of a specific antagonist to VEGF can reduce endothelial monolayer permeability by up to $50 \%$, suggesting an important role for VEGF in regulating alveolar-capillary permeability in ARDS. ${ }^{6}$ In contrast to the plasma compartment, however, bioactive VEGF is reduced in the ELF of patients with pulmonary fibrosis, ${ }^{7}$ emphysema, ${ }^{8}$ sarcoidosis, ${ }^{7}$ following lung transplantation, ${ }^{9}$ and ARDS. ${ }^{10}{ }^{11}$
Furthermore, in ARDS the early restoration of ELF VEGF levels was associated with recovery from lung injury. ${ }^{10}$ It has been suggested that VEGF within the lung may therefore play a dual role, not only in contributing to the regulation of alveolar-capillary permeability but also promoting lung repair. ${ }^{3}$ This hypothesis is supported by recent in vitro studies that have shown that VEGF can enhance alveolar cell proliferation after acid injury ${ }^{12}$ and increase surfactant production from type II cells. ${ }^{1}$

The mechanisms responsible for regulating VEGF in the lung are currently unclear. Several hypotheses have been proposed to explain the low intrapulmonary levels of VEGF. These include decreased production due to the loss of alveolar type II cells, increased degradation of VEGF by proteases released from neutrophils and other inflammatory cells, or increased release of VEGF from the lung to the plasma as a consequence of damage to the alveolar-capillary barrier. ${ }^{3}$ The recent discovery of specific and non-specific cytokine antagonists in patients with ARDS has considerably enhanced our understanding of the complex regulation of the biological effects of proinflammatory cytokines within the alveolar space in ARDS. ${ }^{13}$

We hypothesised that the presence of an antagonist to VEGF in BAL fluid may be important in regulating the bioactivity of VEGF. Soluble VEGFR-1 (sVEGFR-1) is a splice variant of the membrane bound VEGF receptor VEGFR-1. ${ }^{14}$ It was initially isolated from human umbilical vein endothelial cells and is secreted from monocytes, myocytes, and placental tissue. sVEGFR-1 contains the ligand binding domain of the membrane bound receptor and is a potent antagonist of bioactive VEGF. ${ }^{15}$ sVEGFR-1 regulates the bioactivity of VEGF

Abbreviations: ALl, acute lung injury; ARDS, acute respiratory distress syndrome; ELF, epithelial lining fluid; LIS, lung injury score; TNF $\alpha$, tumour necrosis factor $\alpha$; VEGF, vascular endothelial growth factor 
function by forming heterodimers with it which prevent binding and activation of the VEGF receptors. ${ }^{16}$

We hypothesised that the reduced bioactive VEGF found in patients with ARDS might be due to the presence of sVEGFR1. To examine this hypothesis we measured sVEGFR-1 and total VEGF and, as in previous studies, bioactive VEGF in the BAL fluid of patients at risk for ARDS and those with established ARDS. Our second goal was to investigate the relationship between these measures and indices of the severity of lung injury and clinical outcome in patients with established ARDS.

\section{METHODS \\ Subjects}

Patients were studied within 48 hours of admission to the intensive care units of Birmingham Heartlands Hospital (Birmingham, UK) and Southmead Hospital (Bristol, UK) between 2001 and 2003. The study was approved by the local research and ethics committees at each institution.

Patients were identified as having ALI or ARDS according to the American-European consensus statement. ${ }^{17}$ Patients who did not fulfil these criteria but had predisposing risk factors for ARDS were defined as "at risk". Patients were ventilated using pressure controlled ventilation aiming for tidal volumes of $6 \mathrm{ml} / \mathrm{kg}$. Age/sex matched health volunteers (non-smoking, free from respiratory disease) were defined as normal. Bronchoscopy and bronchoalveolar lavage (BAL) was performed in all patients immediately following inclusion and, when possible, 4 days later. The Acute Physiology and Chronic Health Evaluation (APACHE) II score $^{18}$ and Simplified Acute Physiology Score (SAPS) $\mathrm{II}^{19}$ were calculated as a global assessment of the severity of illness in each group. Murray lung injury score (LIS) and $\mathrm{PaO}_{2}$ to $\mathrm{FiO}_{2}$ ration were calculated as a measure of the severity of lung injury.

\section{Bronchoscopy}

Briefly, the bronchoscope was wedged into to a subsegmental bronchus in the middle lobe and $150 \mathrm{ml} 0.9 \%$ saline was instilled in three $50 \mathrm{ml}$ aliquots. The BAL fluid was aspirated and placed immediately on ice until processing. Arterial blood was collected simultaneously into lithium heparin tubes (Becton Dickinson) and stored on ice until processing.

\section{Sample processing}

The chilled BAL fluid was filtered through a single layer of coarse surgical gauze to remove clumps of mucus and then spun at $500 \mathrm{~g}$ for 5 minutes to pellet the cells. The BAL fluid supernatant was collected and stored at $-80^{\circ} \mathrm{C}$ until analysis. A cell count was performed using a haemocytometer and cells were processed for RNA extraction. RNA was extracted from BAL cells using the RNesay mini kit according to the manufacturer's instructions (Qiagen, UK). RNA was quantified at $260 \mathrm{~nm}$ using a UV wavelength spectrophotometer. Whole blood was spun at $500 \mathrm{~g}$ for 10 minutes, the plasma was removed and stored at $-80^{\circ} \mathrm{C}$ until analysis.

\section{Bioactive VEGF and SVEGFR-1 measurement}

Commercially available ELISA kits were used to measure bioactive VEGF (a bioactive assay detecting free 121 and 165; Quantikine R\&D Systems, UK), total VEGF (Chemicron, USA) and sVEGFR-1 (Bendermedics, Germany) according to the manufacturers' instructions.

\section{VEGF recovery from BAL fluid}

BAL fluid from patients with detectable $(n=12)$ and undetectable $(\mathrm{n}=12)$ sVEGFR-1 were spiked with $200 \mathrm{pg} /$ ml VEGF-A (R\&D Systems) for 30 minutes. Bioactive VEGF was measured by ELISA and the percentage of bioactive VEGF recovery was calculated.

\section{ELF and protein determination}

The urea concentration was determined using a commercially available urea kit (Sigma Diagnostics). The volume of ELF was estimated using the formula: ELF volume $(\mathrm{l})=\mathrm{BAL}$ urea $(\mathrm{mmol} / \mathrm{l}) /$ plasma urea concentration $(\mathrm{mmol} / \mathrm{l}){ }^{21}$ BAL fluid protein was measured using the Lowry method. ${ }^{22}$ The protein permeability index was calculated as plasma protein/ BAL protein $\times 100 .{ }^{23}$

\section{BAL cell culture}

BAL cells were cultured for 24 hours in RPMI at a concentration of $1 \times 10^{6} / \mathrm{ml}$ in the presence of $1 \mathrm{ng} / \mathrm{ml}$ tumour necrosis factor $\alpha(\mathrm{TNF} \alpha), 1 \mu \mathrm{g} / \mathrm{ml}$ lipopolysaccharide (LPS), or control media (RPMI). Supernatants were collected and the concentration of sVEGFR-1 was measured by ELISA.

\section{SVEGFR- 1 RT-PCR}

sVEGFR-1 and the housekeeping control gene GAPDH were amplified by RT-PCR. Primers were synthesised (Alta Bioscience, UK). PCR products were analysed by running through a $3 \%(\mathrm{w} / \mathrm{v})$ agarose gel stained with ethidium bromide (Sigma). Bands were visualised by UVP transilluminator and analysed using the ScionImage software package (ScionImage Corp, USA). The sVEGFR-1 band was sequenced and confirmed as specific for the soluble and not membrane associated receptor.

\section{Statistical analysis}

The presence or absence of detectable sVEGFR-1 between patients with ARDS, those at risk, and normal patient groups were analysed using Fisher's exact test. Linear relations between bioactive VEGF, total VEGF, detectable sVEGFR-1, and severity markers (Murray lung injury score, P:F ratio, APACHE II, SAPS II) were tested using Pearson's correlation coefficient on $\log$ transformed data. Data on differences between receptor positive and receptor negative groups were of a non-normal distribution and were analysed using the Mann-Whitney U test. Data on differences between ELF and plasma sVEGFR-1 were normally distributed and analysed using the paired sample $t$ test. Normality testing was performed using the Shapiro-Wilk test. Data are expressed as median and interquartile range (IQR) or mean (SD). Where a statistically significant difference is present, the mean/median difference and 95\% confidence interval (95\% CI) is presented. Data analysis was performed using SPSS 10.0 for Windows. A $p$ value of $<0.05$ was considered significant.

\section{RESULTS}

\section{Demographic characteristics of patients}

Bronchoalveolar lavage was performed in 41 patients within 48 hours of the onset of ALI/ARDS and a repeat bronchoscopy was performed in 17 patients 4 days after the onset of ARDS. Bioactive VEGF data alone from seven of the patients has been reported previously. ${ }^{10}$ Twelve patients at risk of ARDS and 16 normal volunteers were recruited as controls. The factors predisposing the "at risk" group to ARDS were sepsis $(n=7)$, trauma $(n=1)$, and pneumonia $(n=4)$. Three of the "at risk" patients had pneumonia and a P:F ratio of $<40 \mathrm{kPa}$ but only unilateral infiltrates on the chest radiograph. Lavage was performed on the contralateral side to the infiltrates in these patients. Detailed demographic characteristics of the patients are shown in table 1. The mean age of the normal volunteers was 62 (range 25-77).

\section{sVEGFR- 1 in ELF of ARDS patients}

sVEGFR-1 was detectable in the BAL fluid of $48 \%$ (20/41) of patients with ALI/ARDS on day 1 (0-48 hours) (ELF concentration range $1.4-54.8 \mathrm{ng} / \mathrm{ml}$ ) compared with $8 \%$ 
Table 1 Demographic characteristics of patients (according to GOCA stratification ${ }^{24}$ )

\begin{tabular}{|c|c|c|c|}
\hline & $\begin{array}{l}\text { ARDS } \\
(n=41)\end{array}$ & $\begin{array}{l}\text { At risk } \\
(n=12)\end{array}$ & p value \\
\hline \multicolumn{4}{|l|}{ Gas exchange } \\
\hline Mean (SD) P:F ratio ( $\mathrm{kPa})$ & $16.8(8.2)$ & $39.9(10.4)$ & 0.001 \\
\hline $\mathrm{PaO}_{2} / \mathrm{FlO}_{2}>40 \mathrm{kPa}$ & 0 & 9 & \\
\hline $\mathrm{PaO}_{2} / \mathrm{FlO}_{2} 26.8-40 \mathrm{kPa}$ & 14 & 2 & 0.001 \\
\hline $\mathrm{PaO}_{2} / \mathrm{FlO}_{2} 13.46-26.8 \mathrm{kPa}$ & 18 & 1 & \\
\hline $\mathrm{PaO}_{2} / \mathrm{FlO}_{2}<13.46 \mathrm{kPa}$ & 9 & 0 & \\
\hline Spontaneous breathing, no PEEP & 0 & 0 & \\
\hline Assisted breathing, PEEP 0-5 $\mathrm{cm} \mathrm{H}_{2} \mathrm{O}$ & 14 & 8 & NS \\
\hline Assisted breathing, PEEP $6-10 \mathrm{~cm} \mathrm{H}_{2} \mathrm{O}$ & 18 & 2 & \\
\hline Assisted breathing, PEEP $>10 \mathrm{~cm} \mathrm{H}_{2} \mathrm{O}$ & 9 & 1 & \\
\hline \multicolumn{4}{|l|}{ Organ failure (non-pulmonary) } \\
\hline 0 organ & 2 & 2 & \\
\hline 1 organ & 22 & 6 & NS \\
\hline 2 organs & 14 & 2 & \\
\hline 3 organs & 3 & 0 & \\
\hline \multicolumn{4}{|l|}{ Cause } \\
\hline Unknown & 0 & 0 & \\
\hline Indirect lung injury & 17 & 8 & NS \\
\hline Direct lung injury & 24 & 4 & \\
\hline \multicolumn{4}{|l|}{ Other parameters } \\
\hline Age & 62 (18-93) & 68 (29-78) & NS \\
\hline APACHE ॥ & $22.1(9.3)$ & $21.23(12.2)$ & NS \\
\hline SAPS ॥ & $42.6(15.8)$ & $42.8(14.3)$ & NS \\
\hline LIS & $2.7(0.6)$ & $1.15(0.7)$ & 0.01 \\
\hline Mortality & $51 \%$ & $46 \%$ & NS \\
\hline
\end{tabular}

$(1 / 12)$ at risk patients $(p=0.017)$ and none $(0 / 16)$ of the normal controls $(p=0.002)$. By day 4 sVEGFR-1 was detectable in only two out of 18 patients in the ARDS group $(p=0.008$, fig 1$)$. In ARDS patients with detectable sVEGFR$\mathrm{l}$ on day $\mathrm{l}$ there was a positive correlation with the P:F ratio $(r=0.466, \mathrm{p}=0.038)$ and a negative correlation with LIS $(r=-0.430, \mathrm{p}=0.058)$. There were no linear relations with APACHE II or SAPS II scores. The survival of ARDS patients with or without detectable sVEGFR-1 on day 1 was similar in both groups ( $R$ negative $52.4 \% ; R$ positive $50 \%$ ).

\section{Total and bioactive VEGF levels in ELF}

Total VEGF levels in ELF were higher than bioactive VEGF levels at day 1 in all groups (table 2). There was a trend towards lower total VEGF levels in the ELF of ARDS patients $(163.7 \mathrm{ng} / \mathrm{ml})$ compared with at risk subjects $(439.1 \mathrm{ng} / \mathrm{ml}$, $\mathrm{p}=0.07)$ and normal controls $(234.9 \mathrm{ng} / \mathrm{ml}, \mathrm{p}=0.41)$. Total ELF levels of VEGF in ARDS patients on day 1 were

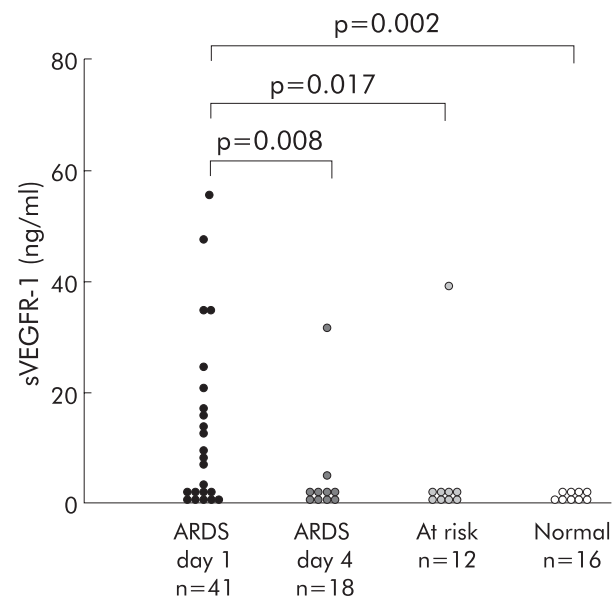

Figure 1 sVEGFR-1 levels in the epithelial lining fluid of patients with ARDS. associated with the APACHE II score $(r=-0.624, \mathrm{p}=0.01)$ and the SAPS II score $(r=-0.554, \mathrm{p}=0.026)$. There was an inverse correlation between total VEGF and protein permeability $(r=-0.675, \mathrm{p}=0.023)$. No difference was found in total ELF levels of VEGF on days 1 or 4 and patient survival.

Consistent with our previous findings, ${ }^{10}$ ELF levels of bioactive VEGF were lower in ARDS patients than in those at risk or normal subjects (table 2). There was a moderate inverse correlation between LIS and bioactive VEGF levels $(r=-0.44, \mathrm{p}=0.01)$. The protein permeability index showed an inverse correlation with bioactive VEGF levels $(r=-0.59$, $p=0.07)$. There was no difference in ELF levels of bioactive VEGF on days 1 or 4 and survival.

ARDS subjects with detectable sVEGFR-1 in ELF had lower median (IQR) levels of bioactive VEGF (1415.2 (474.9$3192.5) \mathrm{pg} / \mathrm{ml}$ ) than those with no detectable receptor (476l (1349.9-7596.6) pg/ml; median difference 3003.9 (95\% CI 305.1 to 4711.9 ), fig 2). There were no linear associations between levels of total or bioactive VEGF and total sVEGFR1. There was a moderate positive correlation between total VEGF and the ratio between bioactive VEGF and sVEGR-1 $(r=0.6 ; \mathrm{p}=0.04)$.

\section{Relationship with total VEGF to sVEGFR-1 ratio}

There was no association between the ratio of total VEGF to sVEGFR- 1 and the LIS $(r=0.215, \mathrm{p}=0.1)$. There were significant inverse relationships between the ratio of total VEGF to sVEGFR-1 and the protein permeability index $(r=-0.625, \quad \mathrm{p}=0.04), \quad$ APACHE II score $(r=-0.6$, $\mathrm{p}=0.01)$, and the SAPS II score $(r=-0.6, \mathrm{p}=0.016)$. There was a non-significant relationship between bioactive VEGF and the ratio of total VEGF to sVEGFR-1 $(r=0.44$, $\mathrm{p}=0.1$ ). There was no difference in the ratio of total VEGF to sVEGFR-1 between survivors and non-survivors.

\section{Plasma levels of sVEGFR- 1 in patients with ARDS}

Mean (SD) ELF concentrations of sVEGFR-1 (8.9 (9.6) ng/ $\mathrm{ml})$ were higher than plasma concentrations $(0.30(0.58) \mathrm{ng} /$ $\mathrm{ml}$ ) in patients with ARDS $(\mathrm{p}=0.001,95 \% \mathrm{CI}$ of difference 
Table 2 Total and bioactive VEGF levels in epithelial lining fluid (ELF)

\begin{tabular}{|c|c|c|c|}
\hline Group & $\begin{array}{l}\text { Median (IQR) total VEGF } \\
(\mathrm{ng} / \mathrm{ml})\end{array}$ & $\begin{array}{l}\text { Median (IQR) bioactive VEGF } \\
\text { (ng/ml) }\end{array}$ & $\begin{array}{l}\text { Median difference } \\
(95 \% \mathrm{Cl})\end{array}$ \\
\hline Normal & $234.9 *(160.6-359.2)$ & $6.52(3.50-9.73)$ & 228.38 (158.6 to 349.6$)$ \\
\hline At risk & $439.1 *(322.7-550.2)$ & $5.01(2.69-8.55)$ & 434.1 (317.8 to 562.7 ) \\
\hline ARDS & $163.7^{*}(77.9-353.8)$ & $2.68+(0.82-5.90)$ & $161.02(107.4$ to 259.6$)$ \\
\hline \multicolumn{4}{|c|}{$\begin{array}{l}\text { * } p<0.001 v \text { ELF levels of bioactive VEGF. } \\
t p<0.05 v \text { ELF levels of bioactive VEGF in at risk and normal subjects. } \\
\text { Bioactive VEGF levels were lower in ARDS patients with detectable sVEGFR-1 in BAL fluid but there was no } \\
\text { difference in total VEGF levels. }\end{array}$} \\
\hline
\end{tabular}

3.9 to $13.5 \mathrm{ng} / \mathrm{ml}$, fig 3). No sVEGFR-1 was detected in the plasma of normal volunteers.

\section{Production of sVEGFR-1 by BAL cells and effect of TNF $\alpha$}

sVEGFR-1 was detected in the BAL fluid supernatants $(\mathrm{n}=10)$ after culture for 24 hours in RPMI (median 1.2 (IQR $0.87-1.61) \mathrm{ng} / \mathrm{ml}$ ). The concentration of sVEGFR-l increased following incubation with TNF $\alpha$ to 1.83 (1.41$2.28) \mathrm{ng} / \mathrm{ml}$, median difference 0.65 (95\% CI 0.05 to 1.256 ), $\mathrm{p}=0.044)$ but not in the presence of LPS $(0.98(0.63-1.38)$, $\mathrm{p}=0.48)$.

\section{Expression of mRNA for sVEGFR-1 in BAL cells}

After finding sVEGFR-1 protein in the BAL fluid supernatants, we looked to see if mRNA for sVEGFR-1 was produced by BAL cells using RT-PCR. sVEGR-1 expression was found in BAL cells from normal subjects, those at risk for ARDS, and ARDS patients ( $\mathrm{n}=14$, fig 4 ). The expression was calculated as a percentage of the housekeeping gene GAPDH to standardise the samples; a range of $31-399 \%$ (mean (SE) $166(23) \%)$ of GAPDH was found, indicating a heterogeneous population. We therefore split the patients into ARDS $(n=5)$, patients at risk of ARDS $(n=4)$ and normal subjects $(n=5)$. The highest mRNA level was found in patients with ARDS (mean (SE) $162(60) \%$ ) compared with the normal group (86 $(13) \%)$ and at risk patients (97 (25)\%), p = 0.1.

\section{Exogenous VEGF in BAL fluid}

Recovery of VEGF spiked into BAL fluid was significantly reduced in BAL fluid from patients with ARDS (recovery 80.1 $(20.5) \%, p<0.001)$. Samples with detectable sVEGFR-1 had lower VEGF recovery than those with none detectable $(72.1 \%$ $v 89.3 \%$ (95\% CI of difference 11.4 to 69.2 ), $\mathrm{p}=0.039$; fig 5 ).

\section{DISCUSSION}

ARDS is characterised by intense inflammation in the alveolar space and the development of a high permeability pulmonary oedema. We initially reported increased levels of bioactive VEGF, a potent endothelial permeability factor, in

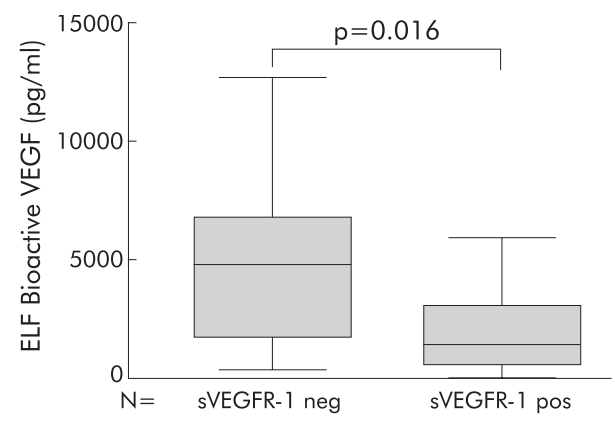

Figure 2 Levels of bioactive VEGF in the epithelial lining fluid (ELF) of patients with and without detectable sVEGFR-1 in BAL fluid. the plasma of patients with ARDS and postulated that VEGF was likely to play a prominent role in the development of non-cardiogenic pulmonary oedema. ${ }^{6}$ Paradoxically, $\mathrm{we}^{10}$ and others ${ }^{11}$ have subsequently found reduced levels of bioactive VEGF in the ELF of patients with ARDS. In the present study the finding of sVEGFR-1, a naturally occurring antagonist to VEGF bioactivity, in the ELF of patients with ARDS suggests a potential mechanism through which the bioactivity of VEGF may be regulated early in its course.

Shortly after the initial descriptions of inflammatory cytokines in the alveolar space in ARDS, specific and nonspecific cytokine antagonists that limit the biological effects of proinflammatory cytokines were discovered. It soon became clear that the net inflammatory balance was of greater physiological and clinical importance than individual cytokine concentrations. ${ }^{1325}$ The tight regulation of the bioavailability of VEGF may be an important factor in the pathophysiology of ARDS. In adult mice transfection with an adenovirus vector containing VEGF 165 led to an increase in the expression of VEGF with a consequential increase in pulmonary capillary permeability and alveolar oedema. However, pretreatment of the mice with an adenovirus vector expressing a truncated soluble form of sVEGFR-1 abrogated completely the development of alveolar oedema in vivo, demonstrating the ability of sVEGFR-1 to effectively inhibit the function of excess VEGF. ${ }^{26}$ We have shown in vitro that plasma from human subjects with ARDS increases human pulmonary artery endothelial cell monolayer permeability by nearly $50 \%$ compared with control plasma. This effect was abolished by the addition of recombinant sVEGFR1 to the plasma of ARDS patients. ${ }^{6}$ It is therefore clear that pulmonary sVEGFR-1 expression early in the course of ARDS may be a physiological response to limit functional increases in the permeability of the alveolar-capillary barrier induced by VEGF.

The novel finding of 50-100 fold higher total VEGF compared with bioactive VEGF in the ELF of patients with

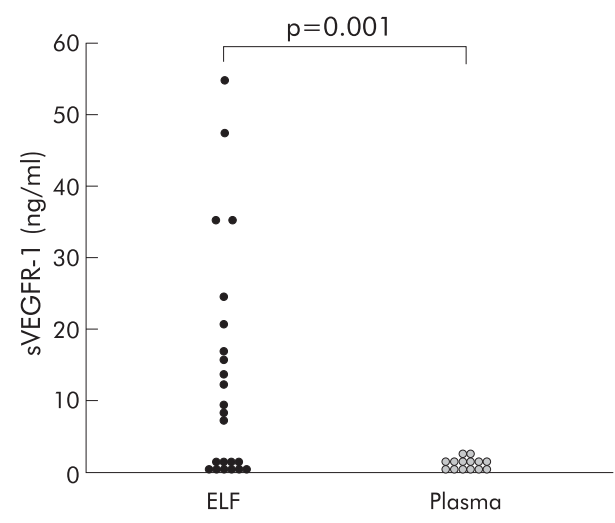

Figure 3 sVEGFR-1 levels in the epithelial lining fluid (ELF) and plasma of patients with ARDS. 


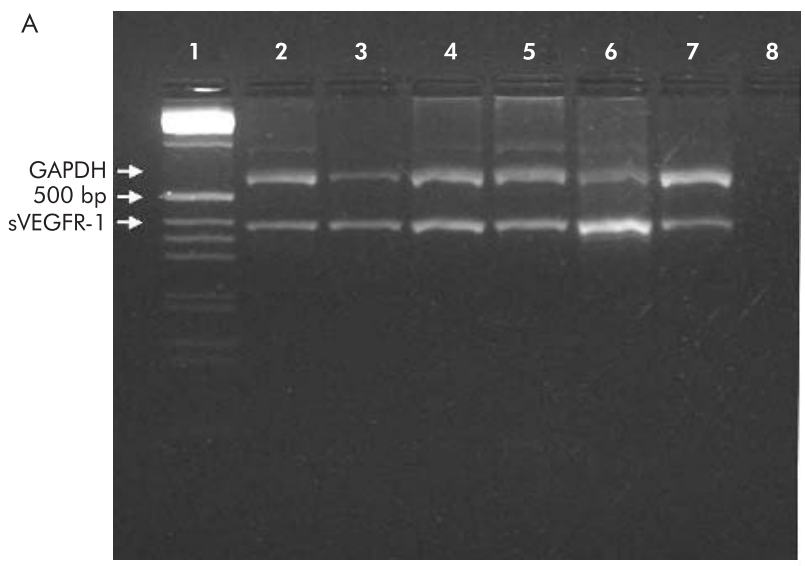

B

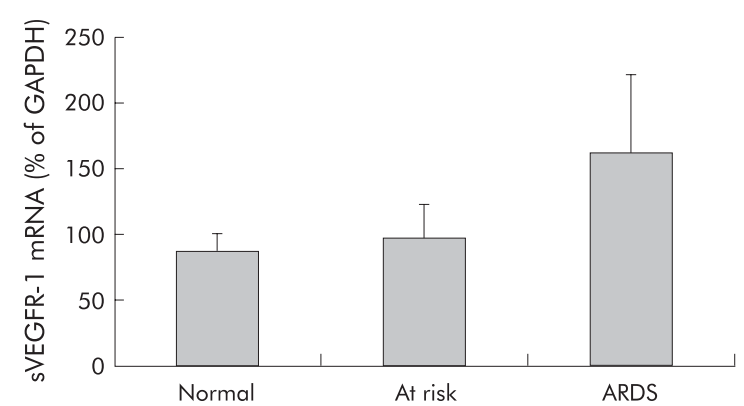

Figure 4 (A) Expression of mRNA for sVEGFR-1 in BAL cells. (A) Lane 1, molecular weight marker (1 kB); lane 2, normal; lane 3, normal; lane 4, at risk; lane 5, at risk; lane 6, ARDS; lane 7, ARDS; lane 8, negative control. (B) mRNA levels in BAL cells from patients with ARDS, those at risk of ARDS and normal subjects.

ARDS supports our hypothesis that regulation of the bioactivity of VEGF is important in ARDS. The presence of sVEGFR- 1 in the ELF of patients with ARDS provides one potential explanation for reduced bioactive VEGF in this patient group. This is supported by the spiking experiments with BAL fluid and VEGF and our finding of a positive correlation between total VEGF and the ratio of bioactive VEGF to total sVEGFR-1. However, only half our patients with ARDS had detectable soluble receptor in the ELF and VEGF recovery was incomplete even in BAL fluid from patients with no detectable sVEGFR-1. One potential explanation could be that the lower limit of detection for the sVEGFR-1 ELISA we used was $80 \mathrm{pg} / \mathrm{ml}$. Consequently, we cannot be sure that the soluble receptor was not present at lower concentrations in our group designated receptor negative.

The local production of SVEGFR-1 in tissues with high VEGF levels has also been reported in placental tissue and primary breast cancer where the ratio of tissue sVEGFR-1 to VEGF has been identified as an independent predictor of outcome. ${ }^{27}$ In parallel with this observation, the findings in the present study of significant correlations between the ratio of sVEGFR-1 to total VEGF and disease severity indices supports our hypothesis that SVEGFR-1 may be important in regulating the biological function of VEGF in ARDS. The finding of mRNA and sVEGFR-1 protein from BAL cell supernatants demonstrates one source for alveolar sVEGFR-1 production in ARDS. This is consistent with our finding of increased levels of sVEGFR-1 in ELF compared with plasma. This apparent compartmentalisation of sVEGFR-1 within the lung may be important for the local regulation of bioactive

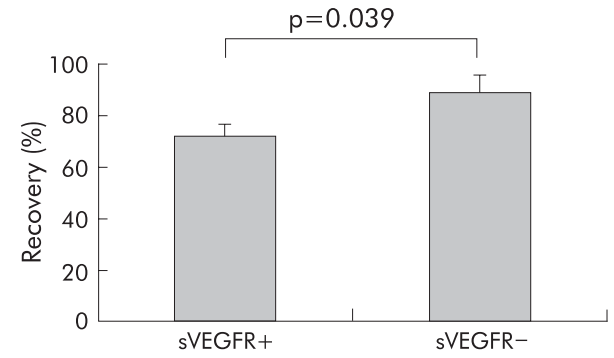

Figure 5 Percentage recovery of VEGF in BAL fluid from patients with ARDS.

VEGF since ELF levels of VEGF are also several times higher in the lung than in plasma.

The disappearance of the soluble receptor from the ELF in all but two patients by day 4 of ARDS suggests that the upregulation of sVEGFR-1 is limited to the early phases of the condition. This is consistent with our previous finding that bioactive VEGF levels tended to increase in resolving lung injury by day $4 .{ }^{10}$ These changes may be important since several studies have recently suggested a beneficial role for VEGF in tissue repair and proliferation. Ohwada has shown that VEGF can restore the ability of acid injured A549 cells (human alveolar cell line) to proliferate. ${ }^{12}$ In animal models of emphysema, blocking the function of VEGF has been shown to result in an increase in markers of oxidative stress, alveolar enlargement, and alveolar cell apoptosis. ${ }^{28} 29$ VEGF also plays an important role in maintaining endothelial cell survival in vitro and in vivo. ${ }^{30}$ The early downregulation of sVEGFR- 1 and the increase in bioactive VEGF may therefore be important for alveolar repair after the initial inflammatory insult. In the present study the number of patients who underwent sequential lavage did not allow us to explore this hypothesis further.

We have not ruled out the possibility that other mechanisms may operate in parallel with sVEGFR-1 production to reduce bioactive VEGF in the lung. There may be other inhibitors to VEGF bioactivity within the lung. ADAMTS1, an extracellular matrix protease, has been shown to inhibit VEGF function by reversibly binding with VEGF and blocking VEGFR-2 phosphorylation leading to the suppression of endothelial cell proliferation in vitro. ${ }^{31}$ Connective tissue growth factor (CTGF) also inhibits $\mathrm{VEGF}_{165}$ binding to VEGFR-2 in vitro by $30 \%$ and reduced the angiogenic effects of $\mathrm{VEGF}_{165}$ in an in vivo mouse angiogenesis model. ${ }^{32}$ Increased VEGF degradation could also explain low levels of VEGF in ELF. Bhattacharjee et al have recently shown that $\alpha_{2}$-macroglobulin, an important proteinase inhibitor, can bind to VEGF resulting in an increase in receptor mediated internalisation and degradation via the $\alpha_{2}$-macroglobulin receptors on macrophages. ${ }^{33}$ Although $\alpha_{2}$-macroglobulin is usually confined to the intravascular compartment, breakdown of the alveolar capillary barrier leads to extravasation of this protein into the alveolar space in $\operatorname{ARDS}^{23}$ where it could potentially stimulate an increase in VEGF degradation.

In our initial study ${ }^{10}$ we hypothesised that the paradox of high plasma VEGF levels but low alveolar levels of VEGF in ARDS could be explained by the loss of VEGF compartmentalisation in the lung due to breakdown of the alveolar capillary barrier. In the present study we investigated the protein permeability index and found an inverse correlation with bioactive VEGF levels. This supports the hypothesis that the increased alveolar-capillary permeability found in ARDS may contribute to the loss of VEGF compartmentalisation within the lung. It is thus possible that the activity and 
function of VEGF within the lung is modulated through several different and independent pathways.

In conclusion, this study has shown a new mechanism for the reduced bioactive levels of VEGF seen in patients with ARDS. sVEGFR-1 levels are higher in the ELF than in plasma in patients with ARDS, suggesting that there is an intrapulmonary source of sVEGFR-1. We also found that BAL cells from patients with ARDS expressed mRNA and produced protein for the receptor, and would appear to be a source for the soluble receptor within the lung. Further research into the source, regulation, and effects of sVEGFR-1 in ARDS is required. It is as yet unclear whether pulmonary sVEGFR-1 expression is the mechanism for reduced bioactive VEGF levels seen in other lung diseases associated with epithelial damage.

\section{Authors' affiliations}

G D Perkins, D F McAuley, F Gao, Intensive Care Unit, Birmingham Heartlands Hospital, Bordesley Green East, Birmingham, UK L Armstrong, A Millar, Lung Research Group, University of Bristol Medical School Unit, Southmead Hospital, Bristol, UK

G D Perkins, J Roberts, D R Thickett, Lung Injury Fibrosis Treatment Programme, University of Birmingham, Lung Investigation Unit, Nuffield House, Queen Elizabeth Hospital, Birmingham, UK

JR was funded by UHB charities. GP was funded by West Medicals Intensive Care Society.

Gene sequencing was supported by BBSRC grant 6/JIF13209.

Conflicts of interest: none declared.

\section{REFERENCES}

1 Compernolle V, Brusselmans K, Acker T, et al. Loss of HIF-2alpha and inhibition of VEGF impair fetal lung maturation, whereas treatment with VEGF prevents fatal respiratory distress in premature mice. Nat Med 2002;8:702-10

2 Kaner RJ, Crystal RG. Compartmentalization of vascular endothelial growth factor to the epithelial surface of the human lung. Mol Med 2001;7:240-6.

3 Mura M, Dos Santos CC, Stewart D, et al. Vascular endothelial growth factor and related molecules in acute lung injury. J Appl Physiol 2004;97:1605-17.

4 Kupferminc MJ, Daniel Y, Englender T, et al. Vascular endothelial growth factor is increased in patients with preeclampsia. Am J Reprod Immunol 1997;38:302-6

5 Thickett DR, Armstrong L, Millar AB. Vascular endothelial growth factor (VEGF) in inflammatory and malignant pleural effusions. Thorax 1999:54:707-10.

6 Thickett DR, Armstrong L, Christie SJ, et al. Vascular endothelial growth factor may contribute to increased vascular permeability in acute respiratory distres syndrome. Am J Respir Crit Care Med 2001;164:1601-5.

7 Koyama S, Sato E, Haniuda M, et al. Decreased level of vascular endothelia growth factor in bronchoalveolar lavage fluid of normal smokers and patients with pulmonary fibrosis. Am J Respir Crit Care Med 2002;166:382-5.

8 Kanazawa H, Asai K, Hirata K, et al. Possible effects of vascular endothelia growth factor in the pathogenesis of chronic obstructive pulmonary disease. Am J Med 2003;1 14:354-8.

9 Meyer KC, Cardoni AL, Xiang Z, et al. Vascular endothelial growth factor in human lung transplantation. Chest 2001;119:137-43.

10 Thickett DR, Armstrong L, Millar AB. A role for vascular endothelial growth factor in acute and resolving lung injury. Am J Respir Crit Care Med 2002; 166:1332-7.
11 Maitre B, Boussat S, Jean D, et al. Vascular endothelial growth factor synthesis in the acute phase of experimental and clinical lung injury. Eur Respir $J$ 2001;18:100-6.

12 Ohwada A, Yoshioka Y, Iwabuchi K, et al. VEGF regulates the proliferation of acid-exposed alveolar lining epithelial cells. Thorax 2003;58:328-32.

13 Park WY, Goodman RB, Steinberg KP, et al. Cytokine balance in the lungs of patients with acute respiratory distress syndrome. Am J Respir Crit Care Med 2001;164:1896-903.

14 Robinson CJ, Stringer SE. The splice variants of vascular endothelial growth factor (VEGF) and their receptors. J Cell Sci 2001:114:853-65.

15 Kendall RL, Thomas KA. Inhibition of vascular endothelial cell growth factor activity by an endogenously encoded soluble receptor. Proc Natl Acad Sci USA 1993;90:10705-9.

16 Ferrara N, Frantz G, LeCouter J, et al. Differential expression of the angiogenic factor genes vascular endothelial growth factor (VEGF) and endocrine gland-derived VEGF in normal and polycystic human ovaries. Am J Pathol 2003;162:1881-93.

17 Artigas A, Bernard GR, Carlet J, et al. The American-European Consensus Conference on ARDS, part 2. Ventilatory, pharmacologic, supportive therapy, study design strategies and issues related to recovery and remodeling. Intensive Care Med 1998;24:378-98.

18 Rowan KM, Kerr JH, Major E, et al. Intensive Care Society's APACHE II study in Britain and Ireland--I: Variations in case mix of adult admissions to general intensive care units and impact on outcome. BMJ 1993;307:972-7.

19 Le Gall JR, Lemeshow S, Saulnier F. A new Simplified Acute Physiology Score (SAPS II) based on a European/North American multicenter study. JAMA 1993;270:2957-63.

20 Bernard GR, Artigas A, Brigham KL, et al. Report of the American-European Consensus conference on acute respiratory distress syndrome: definitions, mechanisms, relevant outcomes, and clinical trial coordination. Consensus Committee. J Crit Care 1994;9:72-81.

21 Rennard SI, Basset G, Lecossier D, et al. Estimation of volume of epithelial lining fluid recovered by lavage using urea as marker of dilution. J Appl Physiol 1986:60:532-8.

22 Lowry OH, Rosebrough NJ, Farr AL, et al. Protein measurement with the folin phenol reagent. J Biol Chem 1951;193:265-75.

23 Holter JF, Weiland JE, Pacht ER, et al. Protein permeability in the adult respiratory distress syndrome. Loss of size selectivity of the alveolar epithelium. J Clin Invest 1986;78:1513-22.

24 Artigas A, Bernard GR, Carlet J, et al. The American-European Consensus Conference on ARDS, Part 2: Ventilatory, pharmacologic, supportive therapy, study design strategies, and issues related to recovery and remodeling. Acute respiratory distress syndrome. Am J Respir Crit Care Med 1998; 157:1332-47.

25 Pugin J, Verghese G, Widmer MC, et al. The alveolar space is the site of intense inflammatory and profibrotic reactions in the early phase of acute respiratory distress syndrome. Crit Care Med 1999;27:304-12.

26 Kaner RJ, Ladetto JV, Singh R, et al. Lung overexpression of the vascular endothelial growth factor gene induces pulmonary edema. Am J Respir Cell Mol Biol 2000;22:657-64

27 Toi M, Bando H, Ogawa T, et al. Significance of vascular endothelial growth factor (VEGF)/soluble VEGF receptor-1 relationship in breast cancer. Int J Cancer 2002;98:14-8.

28 Kasahara Y, Tuder RM, Taraseviciene-Stewart L, et al. Inhibition of VEGF receptors causes lung cell apoptosis and emphysema. J Clin Invest 2000;106:1311-9.

29 Tuder RM, Zhen L, Cho CY, et al. Oxidative stress and apoptosis interact and cause emphysema due to vascular endothelial growth factor receptor blockade. Am J Respir Cell Mol Biol 2003;29:88-97.

30 Ferrara N, Gerber HP, LeCouter J. The biology of VEGF and its receptors. Nat Med 2003;9:669-76.

31 Luque A, Carpizo DR, Iruela-Arispe ML. ADAMTS1/METH1 inhibits endothelial cell proliferation by direct binding and sequestration of VEGF165. $J$ Biol Chem 2003;278:23656-65.

32 Inoki I, Shiomi T, Hashimoto G, et al. Connective tissue growth factor binds vascular endothelial growth factor (VEGF) and inhibits VEGF-induced angiogenesis. FASEB J 2002;16:219-21.

33 Bhattachariee G, Asplin IR, Wu SM, et al. The conformation-dependent interaction of alpha 2-macroglobulin with vascular endothelial growth factor A novel mechanism of alpha 2-macroglobulin/growth factor binding. J Biol Chem 2000;275:26806-11. 